\title{
DIS2015 Heavy Flavours Working Group Summary
}

\author{
Marco Guzzi* \\ Consortium for Fundamental Physics, School of Physics \& Astronomy \\ University of Manchester, Manchester, M13 9PL, United Kingdom \\ E-mail: marco.guzzi@manchester.ac.uk
}

\section{Achim Geiser}

Deutsches Elektronen-Synchrotron DESY,

Notkestrasse 85, 22607 Hamburg, Germany

E-mail: achim.geiser@desy.de

\section{Flera Rizatdinova}

Department of Physics, Oklahoma State University,

145 Physical Sciences Bldg., Stillwater, OK, USA

E-mail: Flera.Rizatdinova@cern.ch

Studies presented in the heavy flavours working group are summarized. Very recent results of measurements at the HERA, LHC, Tevatron, STAR, PHENIX, and BaBar experiments are reviewed and new developments in theory and phenomenology are discussed. In particular, aspects of the impact of heavy flavours on global QCD analyses to determine the structure of the proton, and analyses in physics beyond the Standard Model are considered.

The XXIII International Workshop on Deep Inelastic Scattering and Related Subjects

April 27 - May 1, 2015

Southern Methodist University

Dallas, Texas 75275

\footnotetext{
*Speaker.
} 


\section{Introduction}

Heavy-flavor physics plays a leading role in many areas of collider phenomenology and provides us with powerful tools to handle perturbative and non-perturbative aspects of QCD.

Experimental measurements of cross sections for the production of heavy quarks and different charmed and beauty hadrons, are extremely important to constrain both the structure of the proton in various kinematic regions and fundamental parameters of the Standard Model (SM), such as quark masses and couplings. Precision in the determination of the parton distribution functions (PDFs) of the proton is crucial for the interpretation of measurements in hadronic collisions. PDFs represent a limiting factor in the accuracy of theory predictions for SM processes at the LHC. In this workshop the H1, ZEUS, Tevatron, ATLAS, CMS, and LHCb experiments presented novel measurements which are of high importance for the determination of unpolarized PDFs. Moreover, it has been reported about the progress going on on the theoretical side to reach the new state-of-theart in high-order QCD calculations to determine fully differential cross sections for heavy-flavour production at hadron colliders. Heavy-flavour production (in particular production of top-quark pairs $t \bar{t}$ ) has the potential to impose clean constraints on PDFs (gluon) at large $x$, where they are currently weak.

Heavy-flavour decays were also extensively discussed, in particular new measurements for the B-meson decay and rare charm and beauty-quark decays were presented by the ATLAS, LHCb, and Babar collaborations. Heavy-flavour decays are important to understand the flavour structure of the SM. At the present time, the SM leaves many open questions about the flavour sector: the origin of generation and masses, the mixing and disappearance of antimatter. Investigations on charge and parity (CP) violations in hadronic final states and on rare decays of hadrons made of heavy flavours, offer a powerful way to set constraints on new physics. Furthermore, heavy quarks hadronize in various charmed and beauty hadrons with a large number of possible decays to different final states. This increases the observability of CP violation effects. Therefore, precision measurements of heavy-flavour decays provide us with stringent tests of the Cabibbo-KobayashiMaskawa (CKM) theory $[1,2]$.

A session of the workshop was dedicated to heavy-flavour production in heavy-ion collisions. Heavy-flavour production in high-energy collisions of heavy ions and protons is an excellent probe of the quark-gluon plasma (QGP) state of matter. Investigations of extreme conditions of QCD matter at very high density and temperature open a way to understand the universe in a few millionths of a second after the Big Bang, and to set strong constraints on various model calculations for heavy-flavour QGP interactions. New heavy-flavour production measurements in proton and deuteron heavy-ion collisions were presented by the PHENIX, STAR, and ALICE collaborations.

Many future high-energy physics programs at colliders for the next years will be focussed on the search for any possible signature of physics beyond the SM (BSM). All of the topics presented in the heavy-flavour working group sessions of the DIS2015 workshop are extremely important in this respect. Heavy-flavor physics will play a crucial role in achieving this goal. This was reflected in all experimental and theoretical analyses presented, which triggered a large number of interesting and lively discussions. In what follows, the studies presented in the heavy flavour working group sessions are briefly summarized in chronological order. 


\section{Heavy-flavour production in heavy-ion collisions}

Heavy flavors are suggested as excellent probes to study the properties of the hot and dense nuclear matter created in high-energy heavy ion collisions in the Relativistic Heavy Ion Collider (RHIC) and ALICE experiments. These measurements will be a key to understand evolution of medium effects from proton-proton $(p p)$ to heavy-ion collisions. S. Lim presented measurements $[3,4]$ of the transverse momentum $p_{T}$ of leptons decaying from charmed/beauty hadrons in deuteron + gold $(d+A u)$ collisions at the PHENIX experiment, and discussed several model calculations. Results in Fig.1(left) show suppression of the heavy-flavour pair production at forward rapidity. Z. Ye presented recent results [5] on open heavy-flavor production through semi-leptonic decay channels from the STAR experiment. The results show a strong suppression for the NonPhotonic Electron (NPE) production at $\sqrt{s_{N N}}=200 \mathrm{GeV}$ in gold-gold $(A u+A u)$ collisions. In Fig.1(right) Non-photonic electron azimuthal anisotropies are compared to different model calculations at $\sqrt{s_{N N}}=200 \mathrm{GeV}$. D. Thomas presented measurements [6] of open heavy-flavour production cross sections and their dependence on charged particle multiplicity in $p p(\sqrt{s}=2.76 \mathrm{TeV}$ and $\sqrt{s}=7 \mathrm{TeV})$ and proton-lead $(p+P b)$ collisions $(\sqrt{s}=5.02 \mathrm{TeV})$ at the ALICE experiment. Differences between two-particle correlation distribution in high (0-20\%) and low (60-100\%) multiplicity in the $(\Delta \eta, \Delta \phi)$ space, are shown in Fig.2(left), where a double-ridge structure is observed in heavy-flavour decay electron azimuthal angular correlations in $p+P b$ collisions. K. Kovarik presented a theory talk in which theoretical predictions obtained with shower Monte Carlo programs are compared to different theories where fixed-order calculations are extended with next-toleading logarithms and to predictions using the General Mass Variable Flavour Number Scheme (GM-VFNS). Results [7] are compared to recent measurements of heavy-flavour production in $p p$ collisions at the ALICE experiment [8] in Fig.2(right). These findings are important to understand theory uncertainty for the baseline processes before studying the heavy-quark suppression in heavy-ion collisions.

\section{B-physics and charm/beauty rare decays}

Several presentations focused on the physics of the B-meson and rare decays of charm and bottom-quark decays. B-physics and heavy-flavour rare decays are extremely important as they lead to interplays of the flavour sector and collider physics. Moreover, precise measurements in the flavour sector set severe constraints on new physics. New measurements [9] of B-hadron production with the ATLAS experiment were presented by J. Zalieckas. He showed studies of $B_{c}^{+} \rightarrow J / \psi D_{s}^{(*)+}$ using $\sqrt{s}=7$ and $8 \mathrm{TeV}$ data collected in 2011 and 2012. The main results are shown in Fig.3(left) where branching ratios seem to be generally well described by perturbative QCD (pQCD) and are consistent with several other theoretical models such as QCD potential [10], and Light-front quark model (LFQM) [11]. These results are found to be consistent with similar measurements [12] performed at the LHCb experiment. M. Chrzaszcz presented rare charm and bottom decays measurements [14] using $p p$ collision data at the LHCb experiment. In particular, he discussed the angular analysis of the $B^{0} \rightarrow K^{* 0} \mu^{+} \mu^{-}$decays and results for a relevant angular variable are shown in Fig.3(right). Neglecting the correlations between the observables, the measurements seem to be in agreement with the SM predictions, although the $P^{\prime}{ }_{5}$ angular 

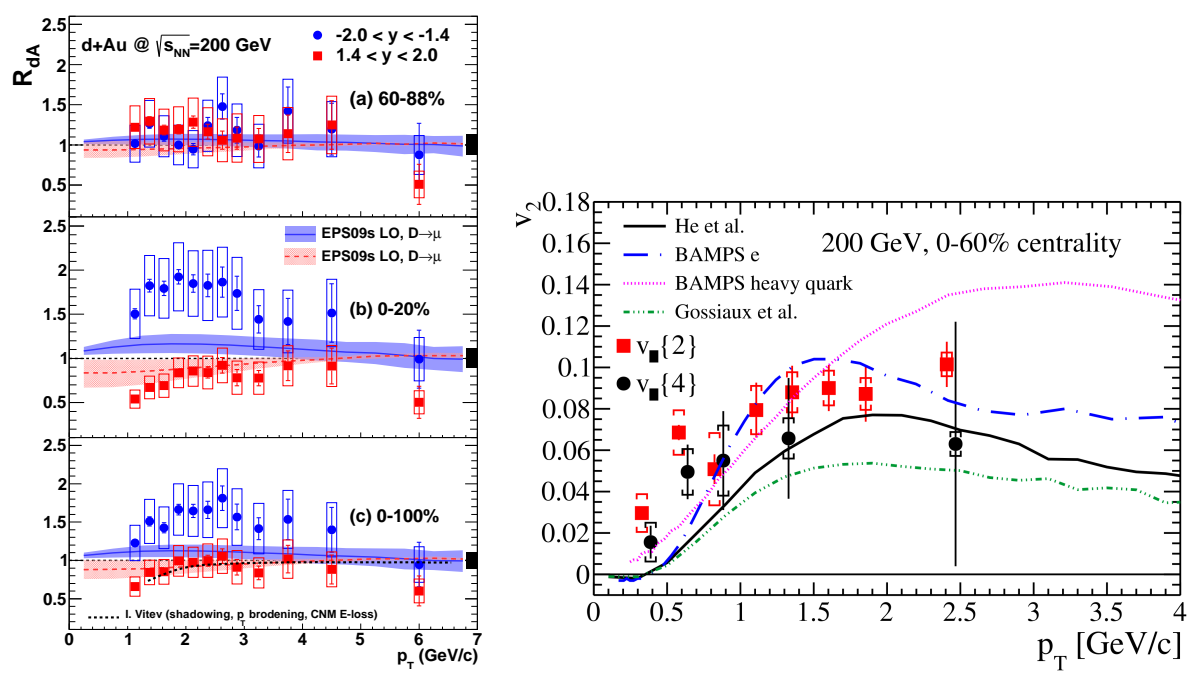

Figure 1: Left: Suppression of the heavy-flavour production at forward rapidity in $d+A u$ collisions at PHENIX. Right: Non-photonic electron azimuthal anisotropies in Au+Au collisions at STAR.
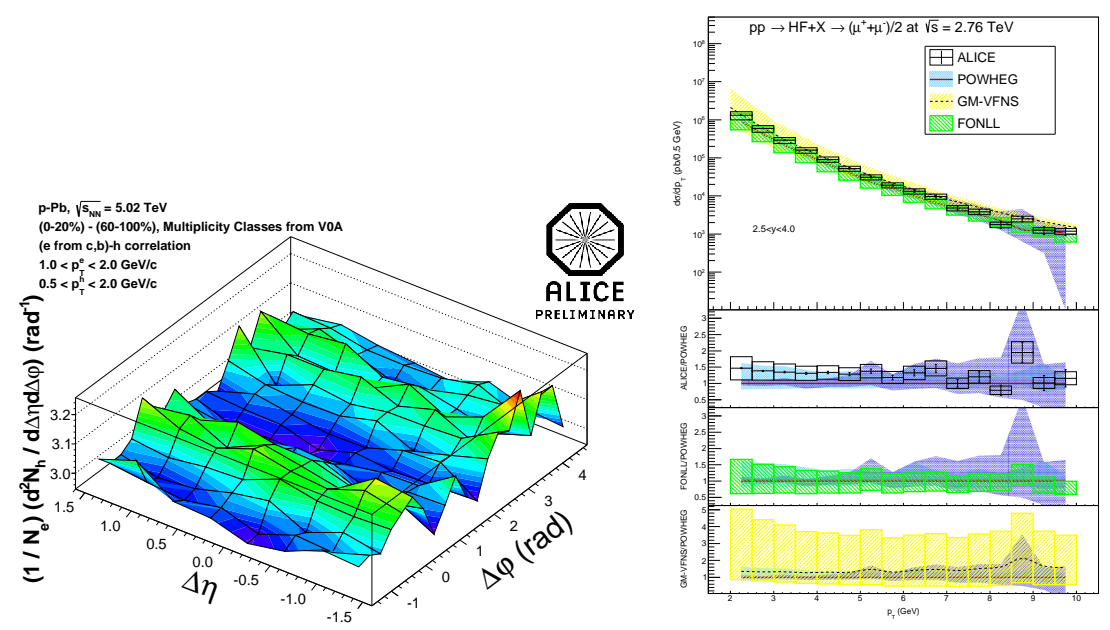

Figure 2: Left: Two-particle correlation distribution in $\mathrm{p}+\mathrm{Pb}$ collisions at ALICE. Right: Transversemomentum distributions of muons from heavy-flavour (charm and bottom-quark) decay produced in the forward region at the LHC with $\sqrt{s}=7 \mathrm{TeV}$ and compared to ALICE data.

observable exhibits a local tension with respect to the SM prediction at a level of $3.7 \sigma$. These results have important implications on the limits on anomalous triple gauge bosons couplings. New theoretical models for B-decays were discussed in M. Ahmady's presentaion [15]. He discussed results obtained from anti-de Sitter Quantum Chromodynamics (AdS/QCD) and used to calculate light cone distribution amplitudes for $\rho$ and $K^{*}$ vector mesons. In Fig.4(left) these distribution amplitudes, utilized to calculate the $B \rightarrow K^{*} \mu^{+} \mu^{-}$differential decay width, are compared to recent $\mathrm{LHCb}$ data. $\mathrm{CP}$ asymmetries between same-sign inclusive dilepton samples $l^{+} l^{+}$and 

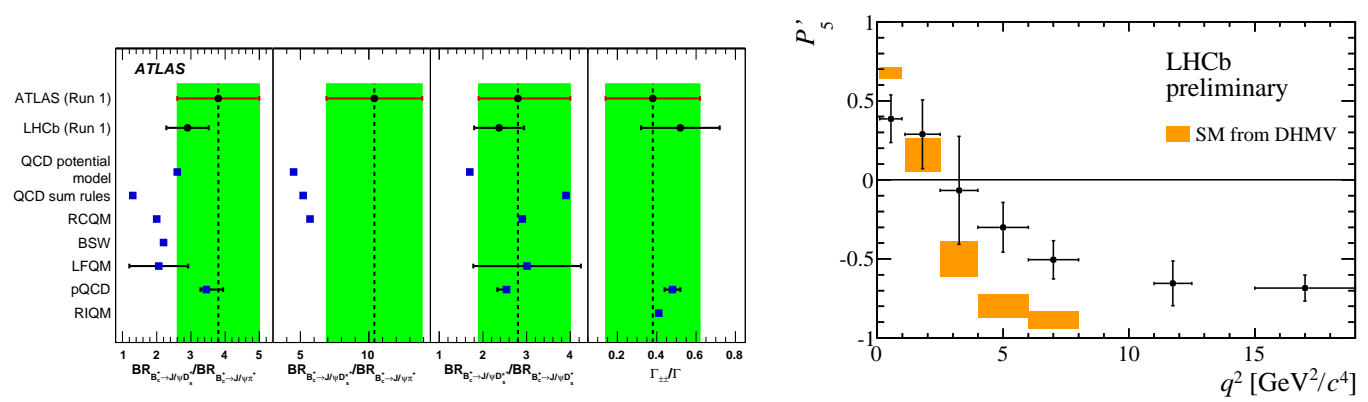

Figure 3: Left: Comparison of the results of this measurement with those of LHCb and theoretical predictions based on a QCD relativistic potential model. Right: The observable $P^{\prime}{ }_{5}$ in bins of $q^{2}$. The shaded boxes show the SM prediction of Ref. [13]
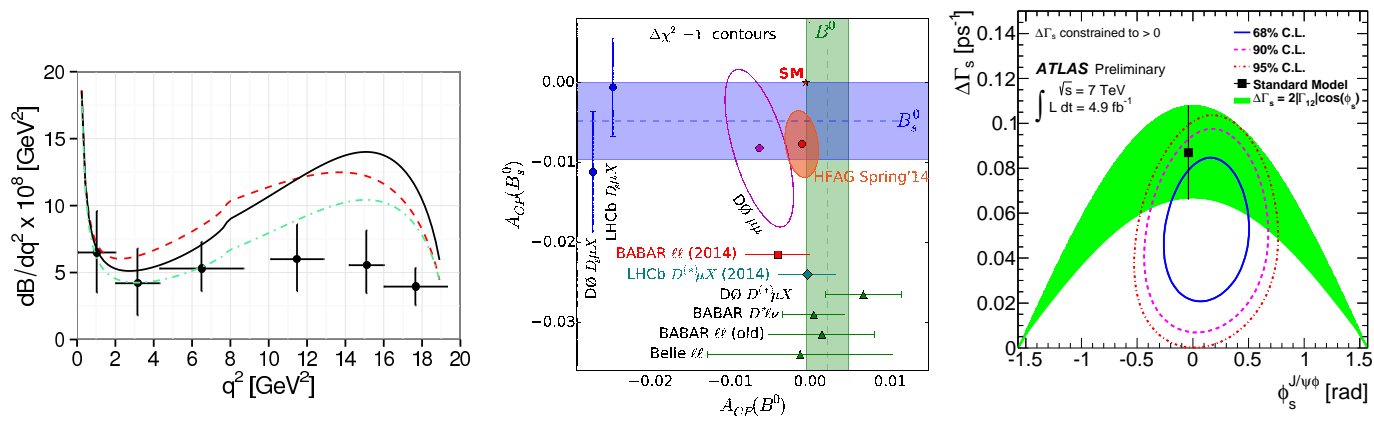

Figure 4: Left: The AdS/QCD prediction for $B \rightarrow K^{*} \mu^{+} \mu^{-}$differential decay width. Center: Measurements of CP asymmetry in neutral B mixing, including this measurement, recent LHCb result. Right: Likelihood contours in $\phi_{s}-\Delta \Gamma_{s}$ plane. The blue and red contours show the $68 \%$ and $95 \%$ likelihood contours, respectively (statistical errors only). The green band is the theoretical prediction of mixing-induced $\mathrm{CP}$ violation.

$l^{-} l^{-}(l=e, \mu)$ from semileptonic B decays in $\Upsilon(4 S) \rightarrow B \bar{B}$ events have been measured [16] at the $\mathrm{BaBar}$ experiment and presented by $\mathrm{M}$. Chrzaszcz. These asymmetries are important probes for $\mathrm{CP}$ and $\mathrm{T}$ symmetry violations. Results are shown in Fig.4(center) where the CP asymmetry is $A_{C P}=(-3.9 \pm 3.5$ (stat. $) \pm 1.9($ syst. $\left.)\right) \times 10^{-3}$ and is consistent with the SM expectation. T. Nooney presented results of new physics searches with B mesons at the ATLAS experiment [17]. In particular, she discussed the parameters of $B_{s} \rightarrow J / \psi \phi$ decay and showed that they are consistent with the values predicted by the SM. Fig.4(right) shows the main results of this study, where the width difference $\Delta \Gamma_{s}$ of the two $B_{s}^{0}$ and $\bar{B}_{s}^{0}$ mesons is represented in terms of the CP violating phase $\phi_{s}$. Many new physics models can significantly affect $\phi_{s}$, therefore this observable is suitable to search for BSM phenomena.

\section{Charm and beauty quark production and exotic mesons}

The charm and beauty-quark production process is going to become extremely important for future precision programs at colliders. Differential cross sections for the production of charm and 
beauty-quark in QCD jets, or in association with vector bosons, are excellent probes of QCD factorization and also have the potential to constrain PDFs of the proton, if such measurements are sufficiently precise. The ATLAS, CMS, Tevatron, and HERA experiments presented several new results for these observables. P. Gunnellini presented new results for the measurement of four-jet production including two b-quark jets at the CMS experiment [18] at $\sqrt{s}=7 \mathrm{TeV}$. The main results of this analysis are shown in Fig.5(left), where the differential cross sections as a function of the $p_{T}$ of the jet is compared to the SM calculation in different rapidity bins. The SM prediction is found to be in good agreement with the data. P.T. Mastrianni showed recent measurements beauty-quark pair production associated with a vector boson at the CMS $[19,20,21]$ experiment at $\sqrt{s}=7 \mathrm{TeV}$. In particular he discussed the total inclusive cross section for $p p \rightarrow Z(l \bar{l})+b \bar{b} X$ process, illustrated in Fig.5(right), and compared the experimental measurements to several theoretical QCD calculations at the NLO accuracy. Data and theory are in very good agreement within the uncertainties. Measurements of open charm production in deep-inelastic electron-proton (ep) scattering (DIS) at
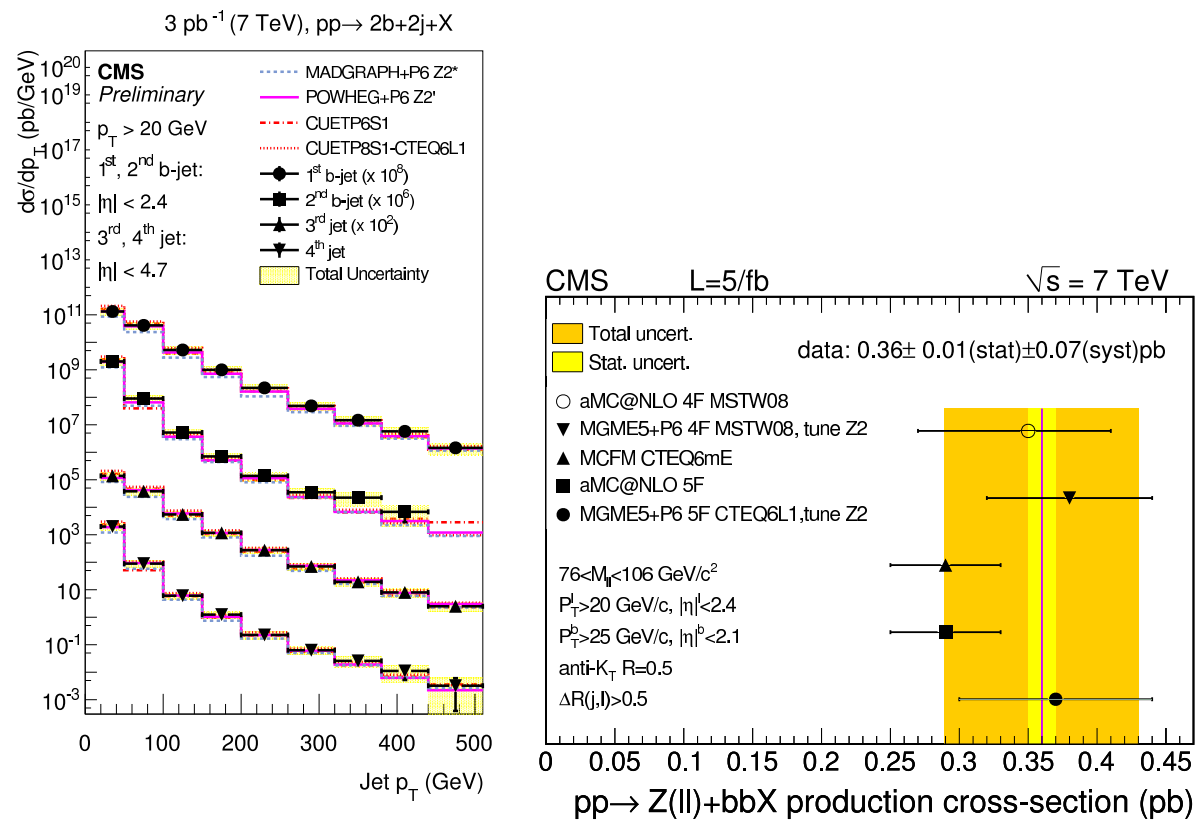

Figure 5: Left: Differential cross sections unfolded to the stable particle level as a function of the jet transverse momenta at CMS $7 \mathrm{TeV}$. Right: Total inclusive cross section for $p p \rightarrow Z(l \bar{l})+b \bar{b} X$ at CMS 7 $\mathrm{TeV}$.

HERA provide important input for stringent tests of the theory of strong interactions. Moreover these precise measurements provide a consistent determination of several important physical quantities such as the charm contribution to the proton structure functions, the charm-quark mass $m_{c}$, and allow us to obtain improved predictions for $W$ and Z-production cross sections at the LHC. Several new results from HERA have been presented in the current workshop. A combination of differential $D^{(*) \pm}$ cross-section measurements from H1 and ZEUS collaborations at HERA [22] was presented by O. Behnke. Perturbative next-to-leading-order QCD predictions are compared to the results for the $p_{T}$ spectrum of the $D^{*}$ in Fig.6(left). The predictions describe the data well 
within their uncertainties, although higher order calculations would be helpful to reduce the theory uncertainty to a level more comparable with the data precision. Further improvements in the treatment of heavy-quark fragmentation would also be desirable. M. Wing presented measurements of $D^{(*)}$ photoproduction at three different centre-of-mass energies from the ZEUS collaboration at HERA [23]. The dependence on the ep centre-of-mass energy was presented for the first time. Variations of the cross section with centre-of-mass energy are sensitive to the gluon PDF in the proton, as different values of Bjorken $x$ are probed. The main results are shown in Fig.6(right) where the normalised $D^{(*)}$ visible photoproduction cross sections as a function of the $e p$ centre-ofmass energy are compared to NLO QCD theory predictions. Measurements are in good agreement with QCD predictions. B.K. Abbott presented for the first time a new measurement of the forward-
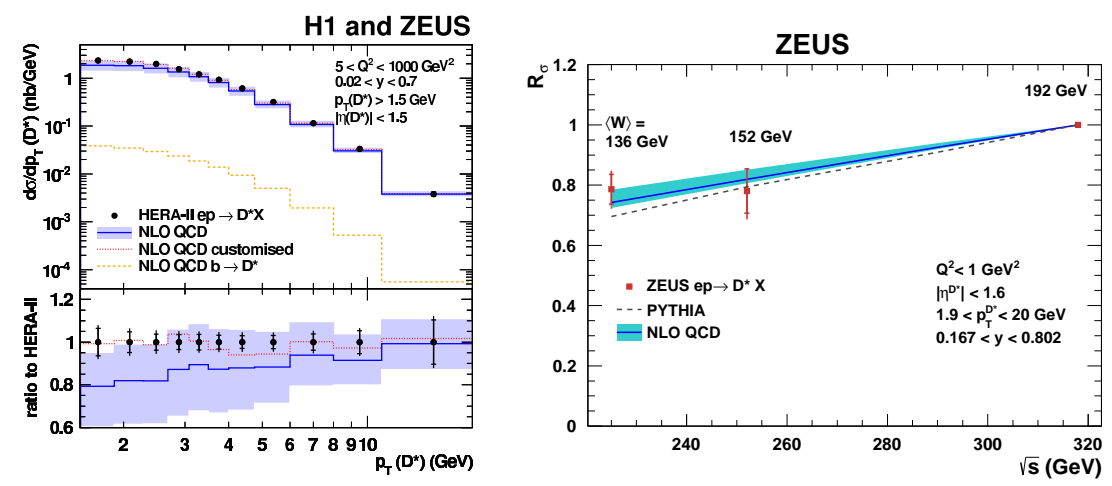

Figure 6: Left: Differential $D^{(*) \pm}$-production cross section as a function of $p_{T}(D)$ at HERA. The data points are the combined cross sections. Right: Normalised $D^{(*)}$ visible photoproduction cross sections as a function of the $e p$ centre-of-mass energy at HERA.

backward asymmetry $\left(A_{F B}\right)$ in the production of $B^{ \pm}$mesons in $p \bar{p}$ collisions at the D0 experiment during the Run-II of the Tevatron collider $[24,25]$. He showed that D0 measured no significant forward-backward asymmetry. The result for $A_{F B}\left(B^{ \pm}\right)=-0.0024 \pm 0.0041$ (stat) \pm 0.0019 (sys) illustrated in Fig. 7(left), is compatible with zero. Locally, some tensions between data and the MC@NLO [26] theory prediction are found. Nonzero asymmetries would indicate a preference for a particular flavor, i.e., b quark or b antiquark, to be produced in the direction of the proton beam. These measurements provide important constraints on the production mechanisms of heavy quarks at hadron colliders.

Searches of exotic states are going on at the LHC. In particular the existence of the $X(3872)$ resonance suggests the presence of its bottomonium counterpart $X_{b}$. J.M. Izen presented a study in which searches for $X_{b}$ states with the ATLAS experiment [27] in final states including $\pi^{+} \pi^{-} \Upsilon$ channel, are discussed. The main results are shown in Fig.7(right), in which exclusion limits for the relative production rate of the $X_{b}$ state are given as a function of its mass.

\section{Top-quark physics: production and properties}

The top-quark physics sessions of the current workshop were very active and triggered several interesting discussions. New results were presented from both experimental collaborations and 

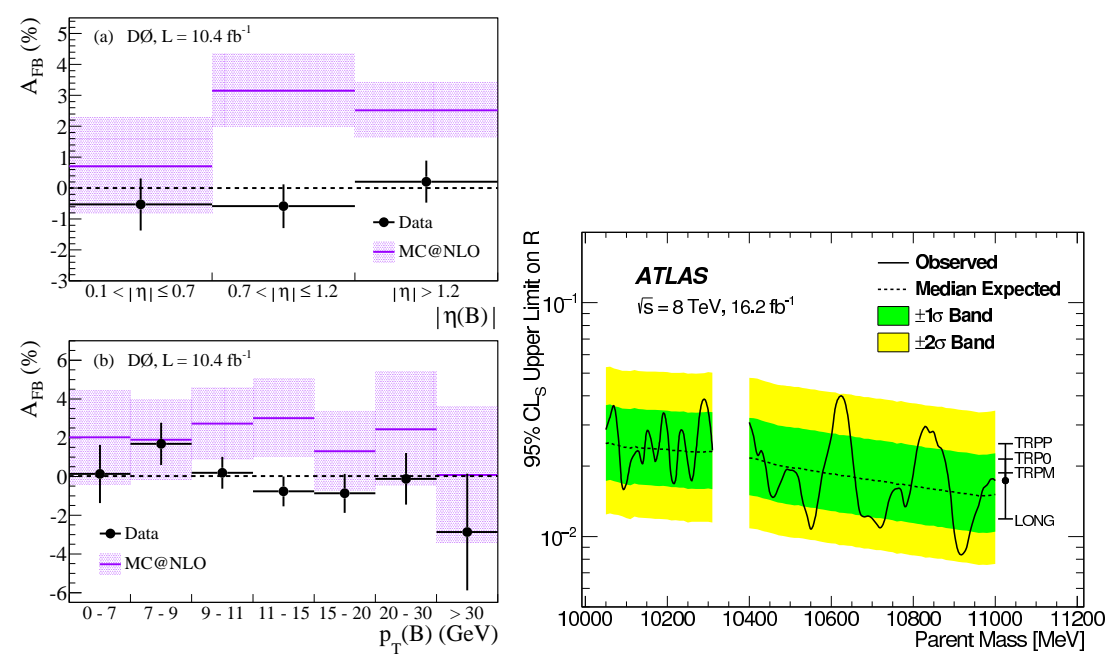

Figure 7: Left: Comparison of $A_{F B}\left(B^{ \pm}\right)$and $A_{F B}^{S M}\left(B^{ \pm}\right)$in bins of $\left|\eta_{B}\right|$ and $p_{T}(B)$. Data points and MC bands include statistical uncertainties convoluted with systematic uncertainties. Right: Observed 95\% CL upper limits (solid line) on the relative production rate of a hypothetical $X_{b}$ parent state decaying isotropically to $\pi^{+} \pi^{-} \Upsilon$, as a function of mass.

theorists. J. Wilson presented new results for the measurements of differential forward-backward asymmetries as a function of rapidity $\Delta y=y_{t}-y_{\bar{t}}$ and invariant mass of bottom- and top-quark pair production at CDF Tevatron [28]. Recent measurements for the $M_{t \bar{t}}$ distribution for $A_{F B}$ are illustrated in Fig.8(left) where these are compared to the POWHEG [29] theory prediction. It has been recently found [30] that the NNLO QCD corrections to the top-quark pair production cross section are large (27\% of the NLO) and when these are combined with the EW (25\% of the NLO) corrections, produce inclusive $A_{F B} \approx 10 \%$. The plot in Fig.8(right), presented by A. Mitov in the plenary session, shows the NNLO QCD prediction for the $M_{t \bar{t}}$ differential asymmetry together with the CDF [31] and D0 [32] measurements. The agreement between the data and theory has improved, and the NNLO theory is less than $1.5 \sigma$ below the CDF data.
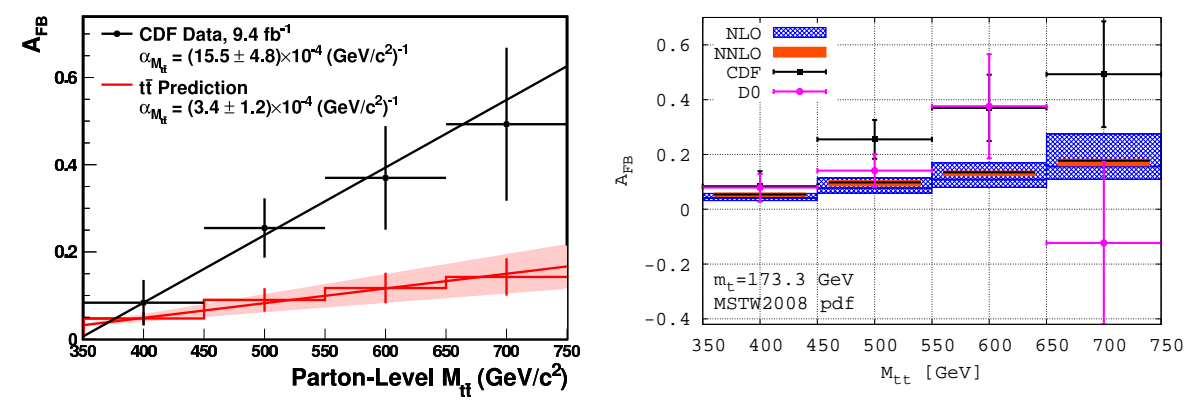

Figure 8: Left: The parton-level $M_{t \bar{t}}$ distribution for the $A_{F B}$ at CDF [31]. The shaded region represents the theoretical uncertainty on the slope of the prediction. Right: $M_{t \bar{t}}$ distribution for the $A_{F B}$ in pure QCD at NLO (blue) and NNLO (orange) versus CDF [31] and D0 [32] data. 
N. Kidonakis reported about the recent progress in approximate QCD calculations for topquark pair production using logarithmic threshold expansions of the resummed cross section. In particular, he showed approximate cross sections up to $\operatorname{NNNLO}\left(\mathscr{O}\left(\alpha_{s}^{5}\right)\right)$ for relevant $t \bar{t}$ observables at the LHC and Tevatron [33]. In Fig.9(left) these higher-order corrections are shown and are compared to recent LHC measurements.
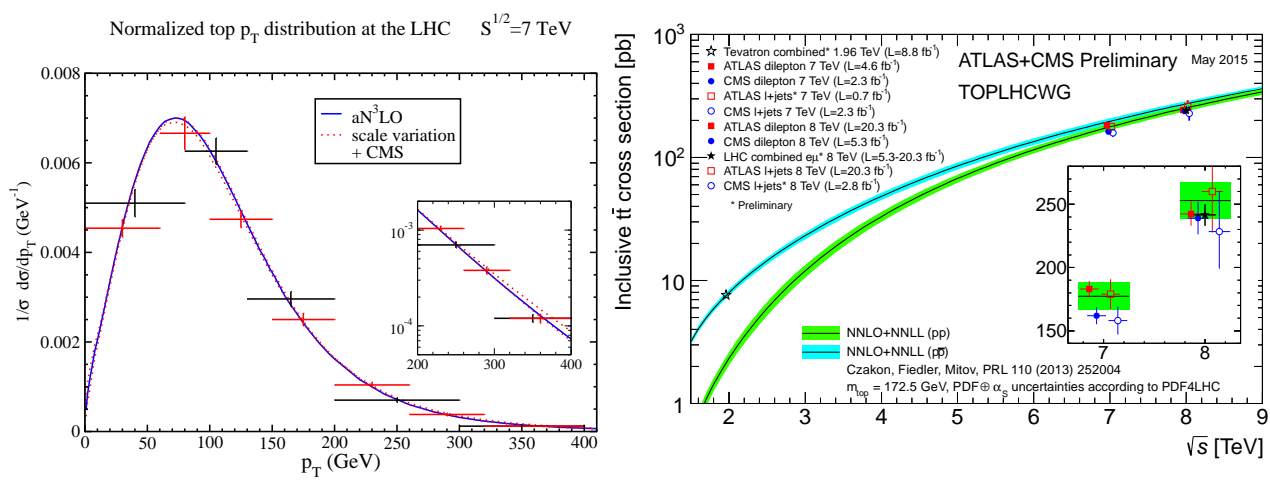

Figure 9: Left: Normalized aN3 LO top-quark pT distributions at the $7 \mathrm{TeV}$ LHC, and comparison with CMS data [34]. Right: Summary of LHC and Tevatron measurements of top-quark pair production.

J.G. Garay, A. Jung, S. Protopopescu, and C. Schwanenberger gave detailed presentations and overviewes on the current status of measurements for top-quark pair production inclusive and differential cross sections at the ATLAS and CMS experiments. C. Schwanenberger summarized results of the measurements of $t \bar{t}+$ jets, $t \bar{t}+\gamma, t \bar{t}+Z$, and $t \bar{t}+W$ production at the ATLAS experiment [35]. Top-quark properties and decays were also extensively discussed. Preliminary ATLAS and CMS combined results for the inclusive $t \bar{t}$ cross section measurements at $\sqrt{s}=8 \mathrm{TeV}$ [36, 37] were presented. The main results are illustrated in Fig.9(right), where LHC and Tevatron measurements of $t \bar{t}$ pair production cross section are shown as a function of the centre-of-mass energy and are compared to the NNLO QCD calculation complemented with NNLL resummation [38]. Examples of measurements of top-quark pair production differential cross sections at ATLAS [39] and CMS [40] at $\sqrt{s}=8 \mathrm{TeV}$, are shown in Fig.10. Precise measurements for these cross sections are extremely important for global QCD analyses to constrain proton PDFs (in particular the gluon) in the large $x$ region, where these are currently poorly determined. Moreover, these measurements provide us with the possibility of pinning down the correlations between the cross section, PDFs, $\alpha_{s}$, and top-quark mass. S. Menke and H. Liu presented measurements of the top-quark mass $m_{t}$ at the LHC and Tevatron respectively. Very recent $m_{t}$ measurements at ATLAS [41] $7 \mathrm{TeV}$ and CMS [42] at $8 \mathrm{TeV}$ are shown in Fig.11, where the CMS summary result of LHC Run-I is $m_{t}=172.38 \pm 0.10$ (stat) \pm 0.65 (sys) GeV. The preliminary ATLAS summary result of the $7 \mathrm{TeV}$ Run-I is $m_{t}=172.99 \pm 0.48$ (stat) \pm 0.78 (sys) GeV. The March 2014 Tevatron plus LHC combined result is $m_{t}=173.34 \pm 0.27$ (stat) \pm 0.71 (sys) GeV. The results presented by $\mathrm{H}$. Liu for the $m_{t}$ combined measurements from CDF and D0 experiments at the Tevatron collider [43] are shown in Fig.11(right). The Tevatron combination resulted in $m_{t}=174.34 \pm 0.37$ (stat) \pm 0.52 (syst) GeV. Measurements of the top-quark properties in the production and decays of $t \bar{t}$ events at CMS [44] and ATLAS [45] were discussed by A. Jung. In particular he discussed measurements of the top-quark 

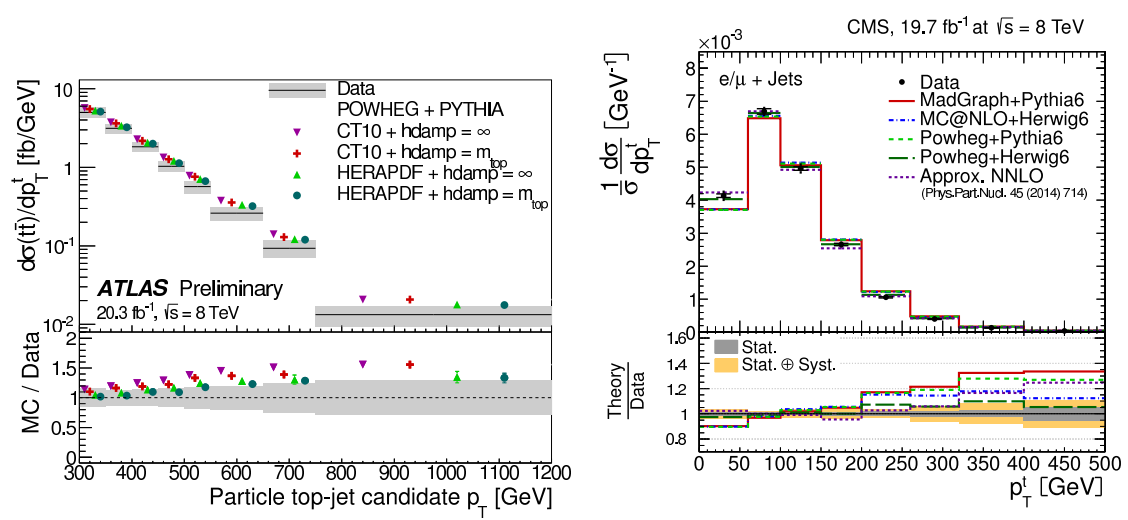

Figure 10: Left: Normalized differential cross-section of highly boosted top quarks as a function of the top quark $p_{T}$. Right: Normalized differential $t \bar{t}$ production cross section in the $l+$ jets channels as a function of the top-quark $p_{T}$

pair charge asymmetry, $W$ helicity in top decays, top-quark charge, $t \bar{t}$ spin correlation, and searches for anomalous couplings. A sample of the main results is shown in Fig.12(left) (ATLAS) and in Fig.12(right) (CMS), where they are compared to the SM predictions and are found to be in agreement. K. Finelli presented measurements of single-top production cross section at ATLAS [46] and CMS [47]. Inclusive and differential cross sections measured in all channels were discussed. The ATLAS and CMS preliminary combination for the total inclusive cross section for single top production is shown in Fig.13(left). Single-top physics is a probe for BSM physics. In fact, single top-quark production cross sections set stringent limits on the mass of extra charged currents decays of $W^{\prime} \rightarrow t b$. Two recent studies are shown at ATLAS [48] and CMS [49] in Fig.13(center, right), where exclusion limits for the mass of right-handed $W^{\prime}$ s are given.

\section{Heavy flavours in global QCD analyses of proton PDFs}

This section is concerned with the impact of heavy flavours in the determination of the structure of the proton in global QCD analyses of world experimental data. Measurements of heavyflavour cross sections play a crucial role in the most recent PDF global analyses [50, 51, 52, 53, 54, 55, 56], and are an essential ingredient of the LHC run-II program [57]. LHC run-II measurements will set a completely new frontier for PDFs accuracy, as they will be constrained in new kinematic regions. Precise determination of PDFs and reduction of their uncertainties are crucial for a correct estimate and characterization of LHC cross sections and for new physics searches. In the studies presented at this workshop, several very recent heavy-flavour cross section measurements have been exploited to constrain PDFs in kinematic regions so far unexplored.

T.J. Hou presented a detailed analyses of the predictions for $g g \rightarrow H$ and $t \bar{t}$ cross sections at the LHC 7, 8 and $13 \mathrm{TeV}$, as well as their uncertainties from both the PDFs and the strong coupling $\alpha_{s}$, in the context of the recent CT14 global QCD analysis [50]. In particular, he discussed a consistency check between the Lagrange multiplier and Hessian method. Some of the main results are shown in Fig. 14, where the behaviour of the $\chi^{2}$ as a function of the total inclusive NNLO $t \bar{t}$ pair 

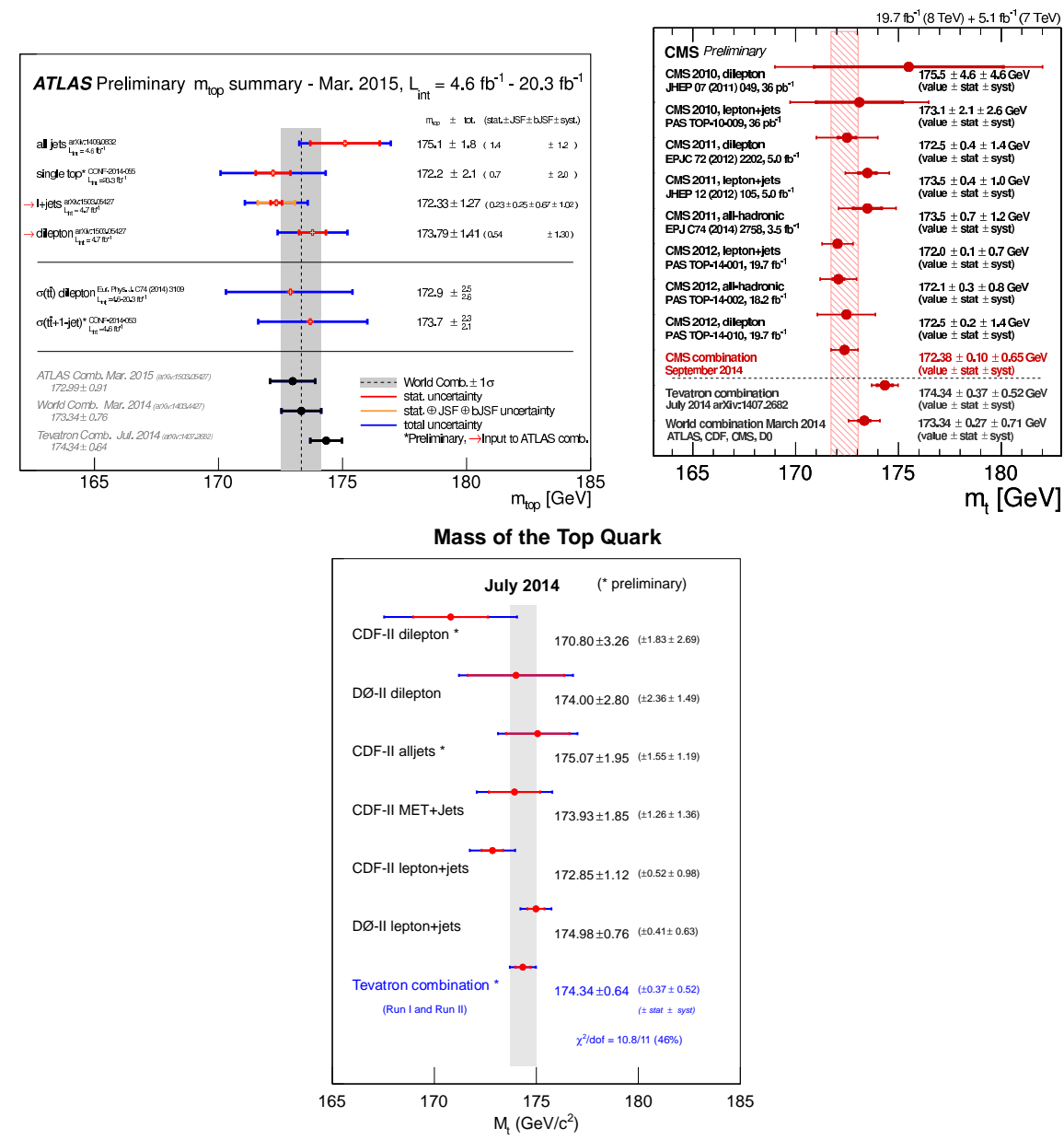

Figure 11: Left: Summary of the latest ATLAS direct $m_{t}$ measurements. Center: Summary of the eight CMS $m_{t}$ measurements and their combination. Right: CDF and D0 $m_{t}$ measurements and their combination.

production cross section (left), and a contour plot (right) of $\Delta \chi^{2}\left(\sigma_{t \bar{t}}, \alpha_{s}\left(M_{Z}\right)\right)$ in the $\left(\sigma_{t \bar{t}}, \alpha_{s}\left(M_{Z}\right)\right)$ plane at the LHC $13 \mathrm{TeV}$, are illustrated. This type of test is extremely important to validate the hessian analysis and check the consistency of PDF uncertainties in kinematic regions where there are no data to set constraints.

A. Geiser presented a novel analysis [58] on behalf of the Prosa collaboration [59], in which recent $\mathrm{LHCb}$ measurements of charm and beauty production cross sections [60, 61], in conjunction with HERA data [62], are used in a NLO PDF analysis to directly constrain the gluon PDF down to $x \approx 5 \cdot 10^{-6}$. This kinematic range is currently not covered by other experimental data in perturbative QCD fits. One of the key differential cross-section measurements for the charmed D0 meson production at the LHCb experiment, is illustrated in Fig.15(left). The main finding of this analysis is illustrated in Fig.15(right), where the gluon PDF is found to be positive and well constrained at $x \approx 10^{-6}$.

G. Brandt discussed an extension of the HERAPDF2.0 QCD fit [53] in which data of charm and jet-production cross section are used to obtain a simultaneous determination of PDFs and 

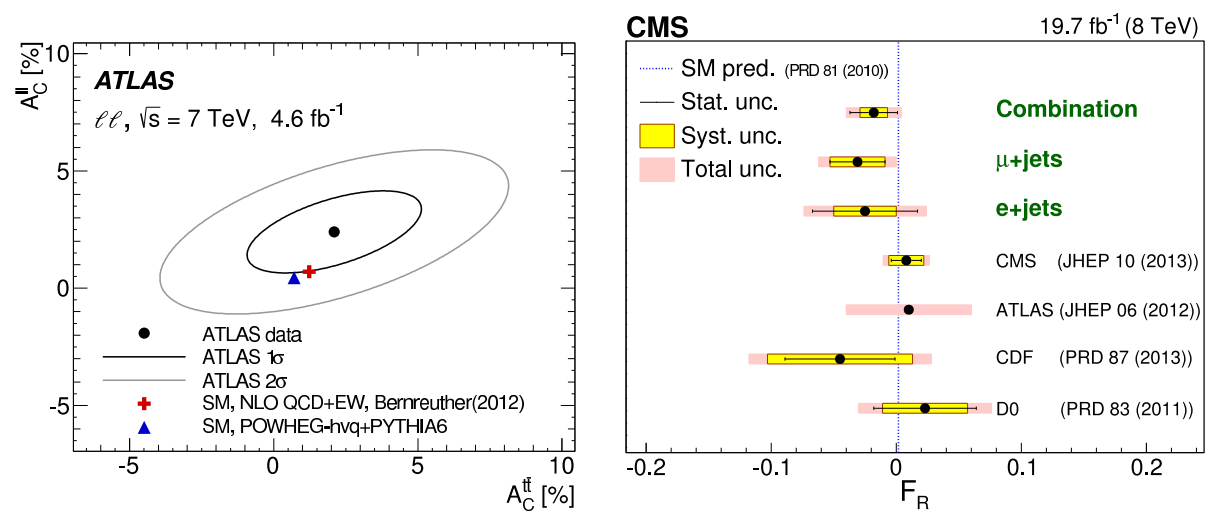

Figure 12: Left: Comparison of the inclusive $A_{C}^{l l}$ and $A_{C}^{t \bar{t}}$ measurement values to the theory predictions (SM NLO QCD+EW prediction). Right: The right-handed helicity fraction of the $W$ boson from the top quark decay.
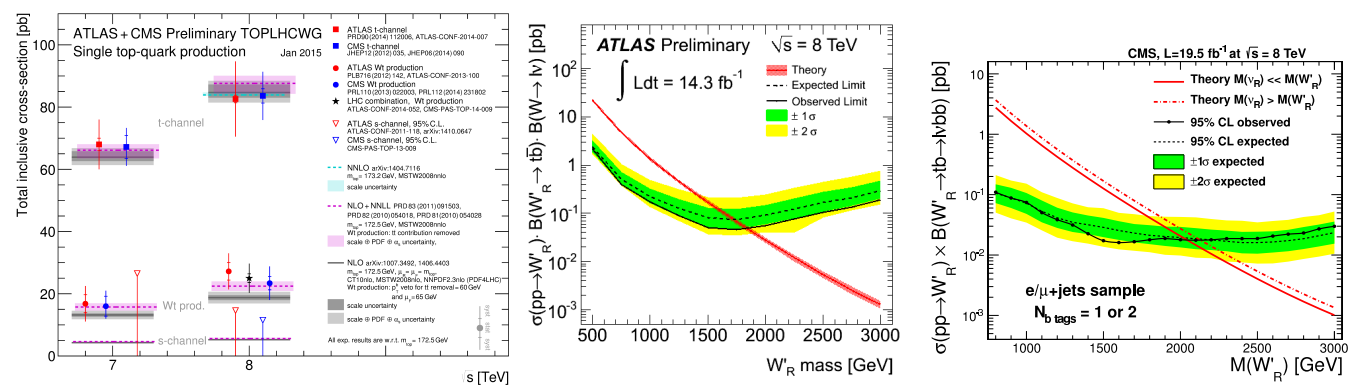

Figure 13: Left: Summary of ATLAS and CMS measurements of the single-top production cross-sections in various channels as a function of the center of mass energy. Center: Observed and expected 95\% CL limits on the $W^{\prime}$-boson cross-section times branching ratio prediction, as a function of the mass of the $W^{\prime}$ boson, for right-handed $W^{\prime}$ bosons at ATLAS $8 \mathrm{TeV}$. Right: Upper limits on the production cross-section of right-handed $W^{\prime}$ bosons obtained for combination of the electron and muon channels at CMS $8 \mathrm{TeV}$.

$\alpha_{s}\left(M_{Z}^{2}\right)$. The new PDFs obtained in this simoultaneous fit are called HERA2.0Jets [53]. The resulting value of the strong coupling constant is $\alpha_{s}\left(M_{Z}^{2}\right)=0.1183 \pm 0.0009(\exp ) \pm 0.0005$ (model $/$ param. $) \pm$ 0.0012 (hadronisation) $+0.0037-0.0030$ (scale). This value is in excellent agreement with the world averge $\alpha_{s}\left(M_{Z}^{2}\right)=0.1185$. In Fig.16(left) shown are different plots of the resulting $\chi^{2}$ for various determinations of $\alpha_{s}\left(M_{Z}^{2}\right)$. In the (right) panel the HERAPDF2.0Jets PDFs at factorization scale $\mu_{f}^{2}=10 \mathrm{GeV}^{2}$ obtained from a NLO fit with free $\alpha_{s}\left(M_{Z}^{2}\right)$, are illustrated. A significant reduction of the uncertainty of the gluon PDF is found.

\section{Conclusions}

Heavy-flavour physics has many implications on the multifaceted aspects of hadronic matter. It is a powerful tool to probe features of QCD in different contexts and it is crucial for searches of new physics. At this conference, a large number of very interesting new results were presented confirming that, even though an impressive amount of important results have already been achieved, 

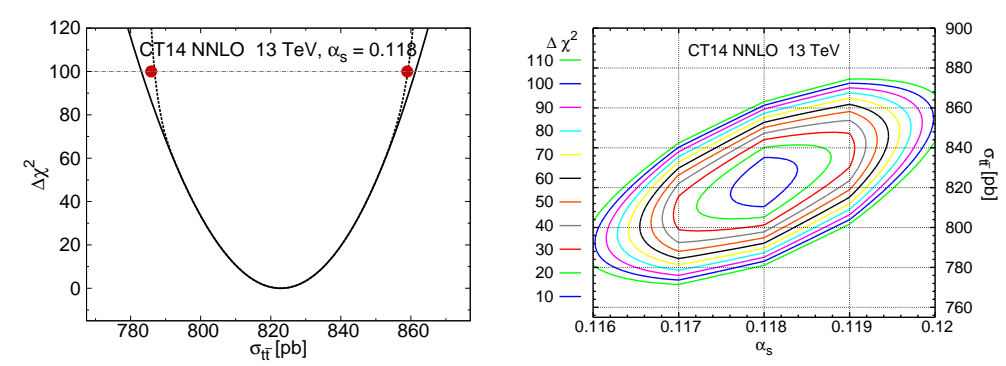

Figure 14: Left: Dependence of the increase in the constrained CT14 fit on the expected cross section at the LHC $13 \mathrm{TeV}$, for $\alpha_{s}\left(M_{Z}\right)=0.118$. Right: Contour plot of $\Delta \chi^{2}\left(\sigma_{t \bar{t}}, \alpha_{s}\left(M_{Z}\right)\right)$ in the $\left(\sigma_{t \bar{t}}, \alpha_{s}\left(M_{Z}\right)\right)$ plane at the LHC $13 \mathrm{TeV}$.
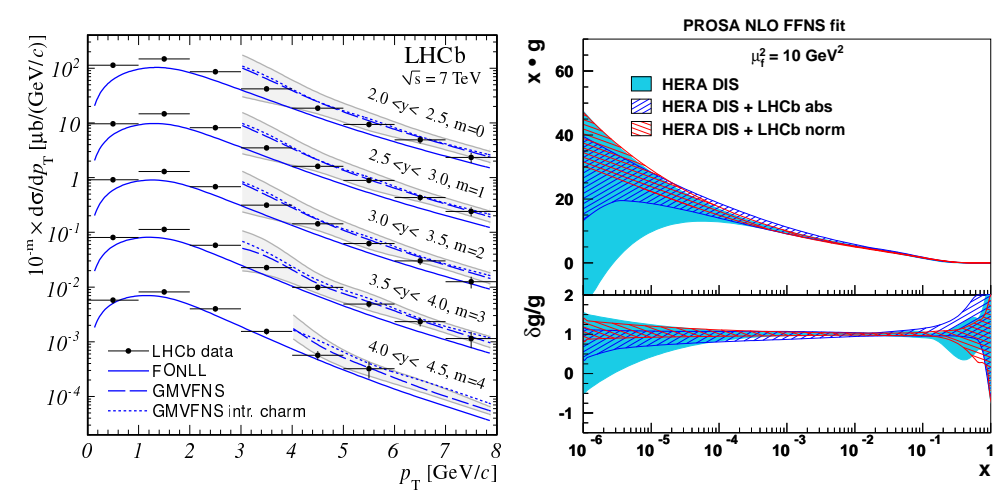

Figure 15: Left: Differential cross-sections for the charmed D0 meson production at the LHCb experiment, compared to NLO theoretical predictions. Right: Gluon PDF uncertainty as obtained from the QCD fit.

a lot of work is ahead to explore key features of QCD and electroweak theory which are crucial to face the challenges of LHC run-II.

\section{Acknowledgments}

This work is supported in part by the Lancaster-Manchester-Sheffield Consortium for Fundamental Physics under STFC grant ST/L000520/1.

\section{References}

[1] N. Cabibbo, Phys. Rev. Lett. 10 (1963) 531.

[2] M. Kobayashi and T. Maskawa, Prog. Theor. Phys. 49 (1973) 652.

[3] PHENIX, A. Adare et al., Phys. Rev. Lett. 112 (2014) 252301, 1310.1005.

[4] PHENIX, A. Adare et al., Phys. Rev. Lett. 114 (2015) 192301, 1404.7461.

[5] STAR, L. Adamczyk et al., (2014), 1405.6348.

[6] ALICE, E.P.d.O. Filho, Proceedings, 6th International Conference on Hard and Electromagnetic Probes of High-Energy Nuclear Collisions (Hard Probes 2013), 2014, 1404.3983. 

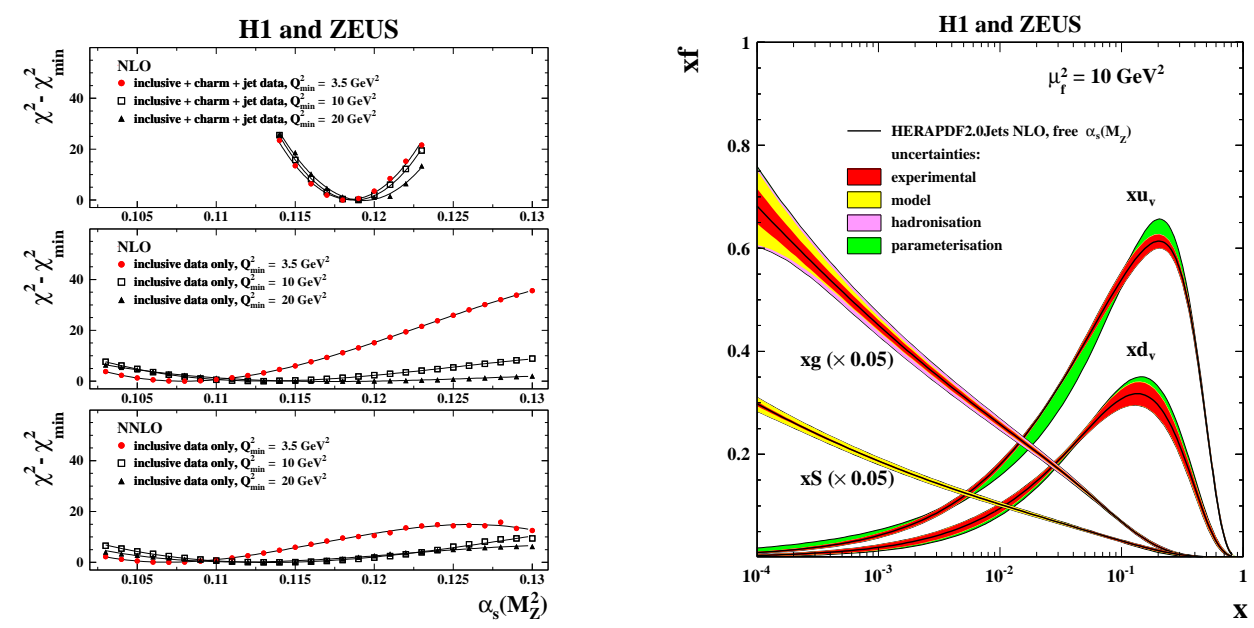

Figure 16: Left: $\Delta \chi^{2}=\chi^{2}-\chi_{\min }^{2}$ vs. $\alpha_{s}\left(M_{Z}^{2}\right)$ for pQCD fits with different $Q_{\min }^{2}$ using data on (a) inclusive, charm and jet production at NLO, (b) inclusive $e p$ scattering only at NLO and (c) inclusive $e p$ scattering only at NNLO. Right: The parton distribution functions $x u_{v}, x d v, x S=2 x(\bar{U}+\bar{D})$ and $x g$ of HERAPDF2.0Jets NLO at $\mu_{f}^{2}=10 \mathrm{GeV}^{2}$ with free $\alpha_{s}\left(M_{Z}^{2}\right)$.

[7] M. Klasen et al., JHEP 08 (2014) 109, 1405.3083.

[8] ALICE, B. Abelev et al., Phys. Lett. B708 (2012) 265, 1201.3791.

[9] ATLAS, G. Aad et al., (2015), 1507.07099.

[10] P. Colangelo and F. De Fazio, Phys. Rev. D61 (2000) 034012, hep-ph/9909423.

[11] H.W. Ke, T. Liu and X.Q. Li, Phys.Rev. D89 (2014) 017501, 1307.5925.

[12] LHCb, R. Aaij et al., Phys.Rev. D87 (2013) 112012, 1304.4530.

[13] S. Descotes-Genon et al., JHEP 12 (2014) 125, 1407.8526.

[14] LHCb, T.L. Collaboration, (2015).

[15] M.R. Ahmady, S. Lord and R. Sandapen, Phys. Rev. D90 (2014) 074010, 1407.6700.

[16] BaBar, J.P. Lees et al., Phys. Rev. Lett. 114 (2015) 081801, 1411.1842.

[17] ATLAS, G. Aad et al., Phys. Rev. D90 (2014) 052007, 1407.1796.

[18] CMS, C. Collaboration, (2015).

[19] CMS, S. Chatrchyan et al., JHEP 06 (2014) 120, 1402.1521.

[20] CMS, S. Chatrchyan et al., Phys. Lett. B735 (2014) 204, 1312.6608.

[21] CMS, S. Chatrchyan et al., JHEP 02 (2014) 013, 1310.1138.

[22] ZEUS, H1, (2015), 1503.06042.

[23] ZEUS, H. Abramowicz et al., JHEP 10 (2014) 3, 1405.5068.

[24] D0, V.M. Abazov et al., Phys. Rev. D91 (2015) 072008, 1503.03917.

[25] D0, V.M. Abazov et al., Phys. Rev. Lett. 114 (2015) 051803, 1411.3021. 
[26] S. Frixione and B.R. Webber, JHEP 06 (2002) 029, hep-ph/0204244.

[27] ATLAS, G. Aad et al., Phys. Lett. B740 (2015) 199, 1410.4409.

[28] CDF, T.A. Aaltonen et al., (2015), 1504.06888.

[29] S. Frixione, P. Nason and G. Ridolfi, JHEP 09 (2007) 126, 0707.3088.

[30] M. Czakon, P. Fiedler and A. Mitov, Phys. Rev. Lett. 115 (2015) 052001, 1411.3007.

[31] CDF, T. Aaltonen et al., Phys. Rev. D87 (2013) 092002, 1211.1003.

[32] D0, V.M. Abazov et al., Phys. Rev. D90 (2014) 072011, 1405.0421.

[33] N. Kidonakis, Phys. Rev. D91 (2015) 031501, 1411.2633.

[34] CMS, S. Chatrchyan et al., Eur.Phys.J. C73 (2013) 2339, 1211.2220.

[35] ATLAS, G. Aad et al., Phys. Rev. D91 (2015) 072007, 1502.00586.

[36] ATLAS, T.A. collaboration, (2014).

[37] CMS, C. Collaboration, (2014).

[38] M. Czakon, P. Fiedler and A. Mitov, Phys.Rev.Lett. 110 (2013) 252004, 1303.6254.

[39] ATLAS, T.A. collaboration, (2014).

[40] CMS, V. Khachatryan et al., (2015), 1505.04480.

[41] ATLAS, G. Aad et al., Eur. Phys. J. C75 (2015) 330, 1503.05427.

[42] CMS, C. Collaboration, (2014).

[43] CDF, D0, T.E.W. Group, (2014), 1407.2682.

[44] CMS, V. Khachatryan et al., JHEP 01 (2015) 053, 1410.1154.

[45] ATLAS, G. Aad et al., JHEP 05 (2015) 061, 1501.07383.

[46] ATLAS, T.A. collaboration, (2014).

[47] CMS, V. Khachatryan et al., JHEP 06 (2014) 090, 1403.7366.

[48] ATLAS, T.A. collaboration, (2013).

[49] CMS, S. Chatrchyan et al., JHEP 05 (2014) 108, 1402.2176.

[50] S. Dulat et al., (2015), 1506.07443.

[51] L.A. Harland-Lang et al., Eur. Phys. J. C75 (2015) 204, 1412.3989.

[52] NNPDF, R.D. Ball et al., JHEP 04 (2015) 040, 1410.8849.

[53] ZEUS, H1, H. Abramowicz et al., (2015), 1506.06042.

[54] S. Alekhin, J. Blumlein and S. Moch, Phys. Rev. D89 (2014) 054028, 1310.3059.

[55] P. Jimenez-Delgado and E. Reya, Phys. Rev. D89 (2014) 074049, 1403.1852.

[56] J. Owens, A. Accardi and W. Melnitchouk, Phys.Rev. D87 (2013) 094012, 1212.1702.

[57] J. Rojo et al., (2015), 1507.00556.

[58] O. Zenaiev et al., (2015), 1503.04581.

[59] https://prosa.desy.de.

[60] LHCb, R. Aaij et al., Nucl.Phys. B871 (2013) 1, 1302.2864.

[61] LHCb, R. Aaij et al., JHEP 1308 (2013) 117, 1306.3663.

[62] ZEUS, H1, F.D. Aaron et al., JHEP 01 (2010) 109, 0911.0884. 